\title{
Measurement of functional and morphodynamic neutrophil phenotypes in systemic inflammation and sepsis
}

\author{
Rens Zonneveld ${ }^{1,2,3^{*}}$ D, G. Molema ${ }^{2}$ and Frans B. Plötz ${ }^{3}$ \\ See related research by Leliefeld et al., http://ccforum.biomedcentral.com/articles/10.1186/s13054-016-1250-4.
}

We read with great interest the review by Leliefeld et al. [1] published in Critical Care on the role of neutrophils in immune paralysis during systemic inflammation (SI). This is of high clinical importance since immune paralysis potentially increases susceptibility to new infections or may cause inability to clear existing infections, leading to detrimental outcome. One mechanism proposed for immune paralysis is the release of neutrophil populations with decreased microbicidal properties [1]. During SI, heterogeneous subsets of neutrophils exist with different priming states and functions [2]. In particular, large numbers of immature neutrophils appear in the circulation with diminished expression of receptors, important for pathogen killing [3]. We recently reviewed the literature on morphodynamic changes in neutrophils during sepsis [4]. Sepsis increases neutrophil circulating numbers (and percentages of immature neutrophils) and their cell size and stiffness, and decreases migration/chemotaxis, compared with nondiseased conditions or mild infection. Septic neutrophils are also prone to produce neutrophil extracellular traps (NETs).

Both reviews $[1,4]$ outline shifting neutrophil identity from an innate responder to a complex immune cell with different functional and morphodynamic phenotypes depending on the underlying pathology. The relationship between changes in functional and morphodynamic phenotypes is not completely understood. For example, the inability of circulating neutrophils to migrate to infectious sites during SI may be the result of diminished expression of chemotactic receptors (e.g., CXCR2), which is most profound in newly released immature granulocytes [1]. Others have suggested a hyperadhesive state of the endothelium, due to overexpression of integrins (e.g., CD11b/ $\mathrm{CD} 18)$ on their membrane, thereby decreasing their ability to migrate [5]. Whether this impaired migration interferes with pathogen killing capacity needs to be investigated.

It is important to know that detecting morphodynamic changes in neutrophils is nowadays clinically feasible with novel technologies [4]. Automated hematology analyzers can measure (immature) neutrophil counts and cell sizes, while microfluidic devices can measure migration velocities. These methods represent a diagnostic toolbox that enables multiparameter analysis of neutrophils during SI and sepsis. When combined with technologies that are still in their infancy (e.g., flow cytometry to measure degranulation), bedside measurement of functions of neutrophils and in-vitro studies as proposed by Leliefeld et al. [1] (e.g., measurement

\footnotetext{
* Correspondence: rens.zonneveld@gmail.com

${ }^{1}$ Department of Pediatrics and Scientific Research Center Suriname,

Academic Hospital Paramaribo, Paramaribo, Suriname

${ }^{2}$ Department of Pathology \& Medical Biology, University Medical Center,

Groningen, University of Groningen, Groningen, The Netherlands

Full list of author information is available at the end of the article
} 
of intracellular or extracellular killing activity) will become feasible. This approach will combine functional and morphodynamic neutrophil phenotypes into clinical settings for better understanding of the exact role of neutrophils in SI and sepsis. Ultimately, this may help to determine whether neutrophils can be considered rational targets for therapy.

\section{Authors' response}

Pieter Leliefeld, Luke Leenen, Janesh Pillay and Leo Koenderman

Numerous studies have focused on the diagnosis and quantification of the innate immune response by measurement of soluble markers (e.g. cytokines, chemokines, bioactive lipids, etc.) in peripheral blood or other body fluids [6]. Particularly, the definition of markers which predict the course of inflammatory complications (e.g., organ failure or secondary infections) induced by trauma and sepsis has gained much attention [7]. Unfortunately, many of these predictive studies failed, merely showing increases in mediators during the clinically most severe period [8]. These measurements therefore lack relevance as predictive markers.

The failure of the abovementioned studies can be explained by the fact that the presence and/or bioavailability of these soluble mediators is affected by many biological processes including differences in clearance rate, binding to natural antagonists, dominant activity of other immunomodulatory mediators, and decoy receptors [8]. There is therefore an increasing interest in utilization of changes in the cellular phenotype of inflammatory cells as a novel diagnostic tool [9], as also discussed by Zonneveld et al. in their letter. This approach assumes that different inflammatory cells integrate all of the proinflammatory and antiinflammatory signals and change their activation state/ phenotype accordingly [9].

A major drawback in the implementation of cellular predictive diagnostics for inflammatory and infectious diseases was the fact that flow cytometry is very labor intensive and difficult to standardize. Recently, the first fully automated flow cytometer, AQUIOS by Beckman Coulter, was developed and is now commercially available [10]. The machine is able to analyze leukocytes in a sample of whole blood in only 20 minutes. In addition, the obtained data can be exported directly to the electronic hospital file system. This allows cellular diagnostics in a 24/7 context with a high reproducibility, as no human action is necessary other than inserting a standardized blood tube in the machine. Preliminary data show that activation of neutrophils in severely injured patients can be monitored by the expression levels of L-selectin (CD62L) and Mac-1 (CD11b) (data not shown).

In conclusion, the concerns raised by Zonneveld et al. for the implementation of cellular diagnostics for inflammatory diseases can be dealt with by application of completely automated flow cytometry in a $24 / 7$ context. We agree that a bedside point-of-care type of analysis is still not possible. However, a report on the status of the innate immune response within 20 minutes is a major step in the immune monitoring of patients at risk for inflammatory complications induced by trauma or infection.

\section{Abbreviations}

CXCR-2, C-X-C motif chemokine receptor 2; NET, neutrophil extracellular trap; SI, systemic inflammation

\section{Funding}

RZ was sponsored by the Thrasher Research Fund.

Authors' contributions

RZ, GM, and FBP conceived, drafted and finalized the manuscript. All authors read and approved the final manuscript.

\section{Competing interests}

The authors declare that they have no competing interests.

\section{Author details}

${ }^{1}$ Department of Pediatrics and Scientific Research Center Suriname, Academic Hospital Paramaribo, Paramaribo, Suriname. ${ }^{2}$ Department of Pathology \& Medical Biology, University Medical Center, Groningen, University of Groningen, Groningen, The Netherlands. ${ }^{3}$ Department of Pediatrics, Tergooi Hospitals, Blaricum, The Netherlands.

Received: 16 May 2016 Accepted: 22 June 2016

Published online: 24 August 2016

\section{References}

1. Leliefeld $\mathrm{PH}$, Wessels $C M$, Leenen LP, Koenderman L, Pillay J. The role of neutrophils in immune dysfunction during severe inflammation. Crit Care. 2016;20(1):73.

2. Pillay J, Ramakers BP, Kamp VM, Loi ALT, Lam SW, Hietbrink F, et al. Functional heterogeneity and differential priming of circulating neutrophils in human experimental endotoxemia. J Leukoc Biol. 2010; 88:211-20.

3. Navarini AA, Lang KS, Verschoor A, Recher M, Zinkernagel AS, Nizet V, et al. Innate immune-induced depletion of bone marrow neutrophils aggravates systemic bacterial infections. Proc Natl Acad Sci U S A. 2009;106:7107-12.

4. Zonneveld R, Molema G, Plötz FB. Analyzing neutrophil morphology, mechanics, and motility in sepsis: options and challenges for novel bedside technologies. Crit Care Med. 2016;44(1):218-28.

5. Blom C, Deller BL, Fraser DD, Patterson EK, Martin CM, Young B, Liaw PC, Yazdan-Ashoori P, Ortiz A, Webb B, Kilmer G, Carter DE, Cepinskas G. Human severe sepsis cytokine mixture increases $\beta 2$-integrin-dependent polymorphonuclear leukocyte adhesion to cerebral microvascular endothelial cells in vitro. Crit Care. 2015;19:149.

6. Xiao Z, Wilson C, Robertson HL, Roberts DJ, Ball CG, Jenne CN, Kirkpatrick AW. Inflammatory mediators in intra-abdominal sepsis or injury-a scoping review. Crit Care. 2015:19:373-86.

7. Gentile LF, Cuenca AG, Vanzant EL, Efron PA, McKinley B, Moore F, Moldawer LL. Is there value in plasma cytokine measurements in patients with severe trauma and sepsis? Methods. 2013;61:3-9.

8. Tetta C, Bellome R, D'Intini V, De Nitti C, Inguaggiato P, Brandolan A, Ronco C. Do circulating cytokines really matter in sepsis? Kidney Int. 2003;84:S69-71. 
9. Hietbrink F, Koenderman L, Althuizen M, Pillay J, Kamp V, Leenen LP. Kinetics of the innate immune response after trauma: implications for the development of late onset sepsis. Shock. 2013;40:21-7.

10. Gossez M, Malcus C, Demaret J, Frater J, Poitevin-Later F, Monneret G. Evaluation of a novel automated volumetric flow cytometer for absolute CD4+ T lymphocyte quantitation. Cytometry B Clin Cytom. 2016. doi:10.1002/cyto.b.21360 [Epub ahead of print]. 